\title{
Correction to: Variability of Some Milk-Associated Genes and Proteins in Several Breeds of Saudi Arabian Camels
}

\author{
Elrashdy M. Redwan ${ }^{1,2}$. Saleh A. Alkarim ${ }^{1} \cdot$ Amr A. El-Hanafy ${ }^{1,3} \cdot$ Yasser M. Saad ${ }^{1,4} \cdot$ Hussein A. Almehdar $^{1}$. \\ Vladimir N. Uversky ${ }^{1,5,6}$
}

Published online: 17 August 2018

○) Springer Science+Business Media, LLC, part of Springer Nature 2018

\section{Correction to: The Protein Journal (2018) 37:333-352 https://doi.org/10.1007/s10930-018-9782-x}

The original version of this article contained mistakes in author names and affiliations. The last names of the authors Salah Korim, Amro Samra, and Hussein A. Amhedar were misspelled. The corrected spelling is Saleh A. Alkarim, Amr A. El-Hanafy, and Hussein A. Almehdar. The correct list of author names and affiliations are published with this erratum.

Elrashdy M. Redwan

redwan1961@yahoo.com; 1radwan@kau.edu.sa

$\triangle$ Vladimir N. Uversky

vuversky@health.usf.edu

1 Biological Sciences Department, Faculty of Sciences, King Abdulaziz University, P.O. Box 80203, Jeddah 21589,

Saudi Arabia

2 Therapeutic and Protective Proteins Laboratory, Protein Research Department, Genetic Engineering and Biotechnology Research Institute, City for Scientific Research and Technology Applications, New Borg EL-Arab 21934, Alexandria, Egypt
3 Department of Nucleic Acid Research, Genetic Engineering and Biotechnology Research Institute, City for Scientific Research \& Technology Applications, Borg EL-Arab 21934, Alexandria, Egypt

4 Genetics Laboratory, National Institute of Oceanography and Fisheries, 101 Kasr Al-Ainy St., Cairo, Egypt

5 Institute for Biological Instrumentation of the Russian Academy of Sciences, Pushchino, Moscow Region, Russia 142290

6 Department of Molecular Medicine and USF Health Byrd Alzheimer's Research Institute, Morsani College of Medicine, University of South Florida, Tampa, FL, USA 